\title{
Specific Cognitive Training Normalizes Auditory Sensory Gating in Schizophrenia: A Randomized Trial
}

\author{
Tzvetan Popov, Todor Jordanov, Brigitte Rockstroh, Thomas Elbert, Michael M. Merzenich, and \\ Gregory A. Miller
}

\begin{abstract}
Background: The ratio of scalp-recorded brain responses occurring $50 \mathrm{msec}$ after paired clicks (S2-evoked P50/S1-evoked P50) serves as a measure of sensory gating. An abnormally large ratio is commonly found in schizophrenia and is considered as a sign of reduced sensory gating or otherwise dysfunctional organization of the auditory/verbal system as a factor contributing to psychopathology and cognitive dysfunction in schizophrenia. This initial randomized clinical trial compared the efficacy of two 4-week, computer-based cognitive training methods that emphasize either auditory discrimination and verbal memory or a broader range of cognitive functions in schizophrenia.
\end{abstract}

\begin{abstract}
Methods: Thirty-nine schizophrenia patients (ICD-F20.0 diagnosis) were assigned to Cognitive Exercises (CE) or Cognitive Package (Cogpack). The M50, the magnetoencephalographic analogue of electroencephalographic P50, and performance on verbal learning and memory tests were used to evaluate training effects.
\end{abstract}

Results: As expected, patients exhibited higher pretreatment gating ratios than 28 age-matched healthy comparison participants. Gating ratios decreased after CE but not after Cogpack. Cognitive test performance improved more after CE than after Cogpack.

Conclusions: Appropriately specific psychological training changes the neural performance in schizophrenia, normalizing sensory and cognitive function.

Key Words: Cognitive training, M50, neuroplasticity, schizophrenia, sensory gating

ognitive deficits are a core feature of schizophrenia (1), and cognitive remediation is an increasingly prominent goal of rehabilitation programs. Yet meta-analyses of cognitive training efforts document small-medium effect sizes for cognitive function outcomes, insufficient stability of effects across time, and limited generalization of trained effects to symptoms or global function (2-4). More efficient strategies have been called for (5). Attempts to overcome past limitations might focus on verbal learning and memory, given that meta-analyses and longitudinal studies have identified these as the most robust abnormalities $(6,7)$. Such attempts might also consider more basic elements of cognitive function, assuming that auditory processing is crucial for the successful encoding and retrieval of verbal information (8) and that increased efficiency in lower-order auditory processes will foster higher-order cognition via more effective engagement of attention and memory processes (9). By training the speed and accuracy of information processing in the cortical auditory system, higher-order cognitive functions such as verbal encoding and memory retrieval should have more precise information input on which to operate (10). Thus, cognitive function should benefit from improved neural signal-to-noise ratio and better discrimination of auditory information.

Training protocols developed with this rationale in mind exploit learning-induced neuroplasticity $(11,12)$ : auditory discrimination and verbal memory tasks are embedded in a suite of graded exer-

From the Department of Psychology (TP, TJ, BR, TE, GAM), University of Konstanz, Konstanz, Germany; Department of Physiology (MMM), School of Medicine, University of California, San Francisco, San Francisco, California; and the Departments of Psychology and Psychiatry and Beckman Institute (GAM), Biomedical Imaging Center, University of Illinois at Urbana-Champaign, Urbana, Illinois.

Address correspondence to Brigitte Rockstroh, Ph.D., Department of Psychology, University of Konstanz, PO Box D23, D-78457 Konstanz, Germany; E-mail: Brigitte.rockstroh@uni-konstanz.de. cises delivered with immediate feedback and an individually adaptive, repetitive practice schedule. Initial evidence indicates improvement on the trained functions and effects on psychopathology and psychophysiological measures $(8,13-15)$. Adcock et al. (13) demonstrated training-induced changes in correlates of the trained function, specifically magnetoencephalographic (MEG) M100 responses to discrimination of successively presented syllables.

The present study used auditory sensory gating, one of the most robust findings of auditory processing abnormalities in schizophrenia $(6,16)$, to explore effects of targeted auditory/verbal discrimination training. Sensory gating refers to the ability of the brain to suppress the response to the second of two paired stimuli. In the auditory modality, sensory gating has usually been studied in a paired-click paradigm: two brief, identical stimuli are presented with 500-msec stimulus onset asynchrony. Whereas both stimuli elicit a response at approximately $50-\mathrm{msec}$ (electroencephalographic event-related potential P50) or MEG M50, the response to the second stimulus is normally attenuated. Thus, the ratio of the $\mathrm{S} 2$-evoked divided by the $\mathrm{S} 1$-evoked $\mathrm{P} 50$ or $\mathrm{M} 50$ represents sensory gating, indicating inhibitory processes as one of the mechanisms that protect processing from irrelevant information (17). Accurate discrimination of information across the stream of consecutive information should support this mechanism, so the gating ratio could also reflect information discrimination fidelity. Variation of $\mathrm{N} 100$ / P200 amplitude with pre-stimulus signal and absolute noise power (18) suggests a relationship to fidelity of sensory information discrimination and neuronal signal-to-noise ratio. Abnormally high auditory sensory gating ratio in schizophrenia patients has been discussed as a sign of impaired sensory filtering (19-21). The functional significance of this basic process for higher cognitive functions is suggested by a relationship between poor P50/M50 gating and neuropsychological dysfunction (attention and working memory) in schizophrenia $(22,23)$. Whether interventions targeting this potentially important neural gateway will have clinical and functional benefits remains to be determined.

In the present study, the effects of a cognitive training protocol targeting discrimination ability in the auditory system (Cognitive Exercises [CE]; PositScience, San Francisco, California) were evalu- 
Table 1. Demographic and Clinical Variables and Demographic, Clinical, and Test Performance Measures

\begin{tabular}{|c|c|c|c|c|c|c|c|c|}
\hline & & \multicolumn{2}{|c|}{ Gender $(\mathrm{m} / \mathrm{f})$} & Age & \multicolumn{2}{|c|}{ Education (yrs) } & \multicolumn{2}{|c|}{ Handedness RH/LH/A } \\
\hline \multirow{3}{*}{\multicolumn{2}{|c|}{$\begin{array}{l}\text { Patients }(n=39) \\
\text { Control Subjects }(n=28) \\
\text { Stat. Difference }\end{array}$}} & \multirow{3}{*}{\multicolumn{2}{|c|}{$\begin{array}{c}35 / 4 \\
20 / 8 \\
x^{2}=2.6, \mathrm{~ns}\end{array}$}} & $30.7 / 8.3$ & \multirow{3}{*}{\multicolumn{2}{|c|}{$\begin{array}{r}12.6 / 2.5 \\
16.7 / 2.2 \\
t(69)=6.9^{\circ}\end{array}$}} & \multirow{3}{*}{\multicolumn{2}{|c|}{$\begin{aligned} & 32 / 4 / 3 \\
& 24 / 3 / 1 \\
& t(65)=-1.2, \mathrm{~ns} \\
&\end{aligned}$}} \\
\hline & & & & $30.1 / 7.4$ & & & & \\
\hline & & & & $t(65)=.3, \mathrm{~ns}$ & & & & \\
\hline & $\begin{array}{c}\text { Gender } \\
(\mathrm{m} / \mathrm{f})\end{array}$ & Age & $\begin{array}{c}\text { Education } \\
\text { (yrs) }\end{array}$ & $\begin{array}{c}\text { Handedness } \\
\mathrm{RH} / \mathrm{LH} / \mathrm{A}\end{array}$ & $\begin{array}{l}\text { Admissions } \\
\text { (n) }\end{array}$ & $\begin{array}{c}\text { Medication } \\
\mathrm{A} / \mathrm{T} / \mathrm{C}\end{array}$ & $\begin{array}{c}\mathrm{CPZ} \\
\text { Pretraining }\end{array}$ & $\begin{array}{c}C P Z \\
\text { Post-Training }\end{array}$ \\
\hline$C E(n=20)$ & $18 / 2$ & $31.16 / 7.8$ & $13 / 2.5$ & $16 / 3 / 1$ & $5.7 / 7.4$ & $15 / 1 / 3$ & $193.32 / 243.8$ & $133.69 / 158.4$ \\
\hline Cogpack $(n=19)$ & $17 / 2$ & $30.26 / 9.2$ & $12.3 / 2.5$ & $16 / 1 / 2$ & $4.7 / 3.9$ & $13 / 3 / 4$ & $144.99 / 180.2$ & $181.87 / 253.5$ \\
\hline \multirow[t]{2}{*}{ Stat. Difference } & $x^{2}=.11, \mathrm{~ns}$ & $t(37)=.3, \mathrm{~ns}$ & $t(37)=.9, \mathrm{~ns}$ & $t(37)=.1, \mathrm{~ns}$ & $t(32)=.5, \mathrm{~ns}$ & $t(35)=.7, \mathrm{~ns}$ & $t(35)=.8, \mathrm{~ns}$ & $t(33)=.8, \mathrm{~ns}$ \\
\hline & $\begin{array}{l}\text { Smoking } \\
\text { Pretraining }\end{array}$ & $\begin{array}{c}\text { Smoking } \\
\text { Post-Training }\end{array}$ & BPRS & GAF & $\begin{array}{c}\text { Immediate } \\
\text { Recall }\end{array}$ & $\begin{array}{l}\text { Working } \\
\text { Memory }\end{array}$ & $\begin{array}{c}\text { Delayed } \\
\text { Recall }\end{array}$ & $\begin{array}{l}\text { Word } \\
\text { Fluency }\end{array}$ \\
\hline $\mathrm{CE}(n=20)$ & $18.8 / 8 / 7.2$ & $18.4 / 7$ & $46.2 / 10$ & $37.3 / 7.5$ & $5.5 / 1.8$ & $11.8 / 2.1$ & $9.8 / 2.9$ & $25.2 / 23.1$ \\
\hline Cogpack $(n=19)$ & $16.6 / 8.6$ & $16.5 / 8.3$ & $52.7 / 15.2$ & $31.7 / 9$ & $5.8 / 1.7$ & $11.5 / 2.6$ & $9.1 / 4.3$ & 20.1/18.9 \\
\hline Stat. Difference & $t(24)=.4, \mathrm{~ns}$ & $t(24)=.5, \mathrm{~ns}$ & $t(32)=1.4, \mathrm{~ns}$ & $t(32)=1.9, \mathrm{~ns}$ & $t(34)=.6, \mathrm{~ns}$ & $t(34)=.4, \mathrm{~ns}$ & $t(34)=.6, \mathrm{~ns}$ & $t(34)=.7, \mathrm{~ns}$ \\
\hline
\end{tabular}

Demographic and clinical variables in patients and control subjects; demographic, clinical, and test performance measures in the two patient subgroups assigned to Cognitive Exercises (CE) and Cognitive Package (Cogpack) treatment groups. Smoking: number of cigarettes/day. Clinical and test performance measures refer to assessment before treatment assignment, if not specified as pre- and post-training. Variables (except for gender) are described by mean and SD.

Handedness A, ambidexter; LH, left-handed; RH, right-handed; Medication A, atypical antipsychotics; T, typical antipsychotics; C, combination of typical and atypical antipsychotics; CPZ, chlorpromazine; BPRS, Brief Psychiatric Rating Scale; GAF, Global Assessment of Functioning. ns, $p>$.1.

${ }^{a} p<.01$.

ated by measuring auditory gating ratios and overt performance on verbal memory and fluency tests before and after training. The CE effects were compared with those of a standard cognitive training regimen (Cognitive Package [Cogpack]; Marker Software, Ladenburg, Germany) addressing a broad range of cognitive features having well-established effects on global cognitive function (2426). This active comparison condition was chosen for similarity with respect to computer-based presentation and a 4-week training period.

\section{Methods and Materials}

\section{Participants}

Inpatients were recruited, evaluated, and treated at the regional Center for Psychiatry. Inclusion criteria were an ICD diagnosis of paranoid-hallucinatory schizophrenia (code number 20.0), age 20-50 years, normal intellectual function, and no history of any neurological condition or disorder including epilepsy or head trauma with loss of consciousness. None of the patients had undergone electroconvulsive treatment. Patients meeting inclusion criteria were informed about the training and measurement protocol and were included in the pretreatment assessment and random assignment protocol after signing written informed consent. All patients were evaluated and trained in a clinically stable state. Sample size reflected eligible patients available January 2008-February 2010. Eleven (5 CE, 6 Cogpack) of 50 patients did not complete the study -6 discharged before the end of the treatment, 3 quitting the treatment early, 1 declining MEG, and 1 providing artifactcontaminated data. The MEG results from the 39 completers are reported here (Supplement 1).

Recruitment and random assignment were done by different staff (BR and TP, respectively). Patients were randomly assigned to the two treatment programs via coin toss. Exceptions were three patients familiar with Cogpack from previous admissions and/or their regular rehabilitation program, who declined to participate in it. These patients were assigned to the CE protocol to avoid dropout. The protocol was continued until groups of sufficient size were assembled. Twenty patients completed $\mathrm{CE}$, and 19 patients completed Cogpack (Table 1). All patients were receiving psychoactive medication (see Table 1 for type and amount). Upon treatment assignment, groups did not differ in gender distribution, age, educational level, global ratings of symptom severity (Brief Psychiatric Rating Scale [BPRS]) [27,28]; Global Assessment of Functioning [GAF]; DSM-IV), number of previous hospital admissions, type or amount of medication, smoking habits, or overt performance on verbal memory and fluency tests.

For evaluation of sensory gating, 28 healthy participants were recruited to be comparable to the patient sample in age and gender (Table 1). Participants were included if they did not meet criteria for a lifetime diagnosis of mental illness (screened with the Mini International Neuropsychiatric Interview interview) (29), did not report any history of head trauma with loss of consciousness, and were free of psychoactive medication. Patients and control subjects did not differ with respect to gender distribution or age, although control subjects had more education (Table 1). For evaluation of sensory gating stability and to provide a comparison group for MEG retesting, 15 of the healthy participants participated in the MEG protocol again after 4 weeks.

Participants gave written informed consent for participation. Participants received $€ 20$ for each 2-hour MEG session. Participation in treatment was introduced as part of the rehabilitation regimen with no additional monetary payment.

\section{Study Design, Cognitive Assessment, and Treatment Protocols}

The study design was approved by the Ethics Committee of the University of Konstanz. Before and after treatment, auditory sensory gating was recorded in a paired-click design (see following), and overt cognitive performance of patients was assessed with a German equivalent of the California Verbal Learning Test (Verbaler Lern-und Merkfähigkeitstest [VMLT]) (30) and a verbal fluency test (Regensburger Wortflüssigkeitstest [RWT]) (31). Based on serial learning of 15-word lists, the VMLT assesses immediate recall, working memory capacity (number of successfully recalled items/list after five repe- 
titions), and delayed recall (after 30 min including the distraction of a second word list). The RWT assesses word production across 2 $\min$.

Patients were assigned to either the auditory-focused CE training program or the broader Cogpack cognitive training program that is currently standard on the inpatient unit for patients who receive such training. The $\mathrm{CE}$ consists of six computerized exercises (8): judging gradually more difficult distinctions between frequency modulation sweeps of auditory stimuli increasing or decreasing in frequency, distinguishing phonemes with synthesized speech, identifying arrays of open and closed syllables in spatial and temporal context, discriminating tone frequencies, and remembering details of a short narrative. Thirteen patients completed the original English version, and 7 completed a German version translated by PositScience, with one verbal memory exercise substituted by additional tone discrimination exercise. Assignment was random, because all patients fully understood exercises and instructions in English. Gating ratios and test performance did not differ for patients trained on the English versus the German version. Cogpack includes a much broader array of 64 exercises of visuomotor skills, vigilance, comprehension, language, memory, logic, and everyday skills. Each Cogpack exercise is available with several variations.

Both treatment protocols were computer-based and adaptive to foster positive reinforcement and avoid failure. Treatment methods were similar with respect to total duration of treatment (4 weeks). The CE comprised 60-min daily sessions on 20 consecutive workdays, whereas Cogpack followed the standard protocol as recommended by the developers: a series of tasks to be accomplished during each of three 60-90-min sessions/week. Treatments were broadly similar in frequency and duration of training sessions and in observed participant effort and tolerance.

\section{Auditory Gating Measurement and Analysis}

In each MEG session, 100 pairs of 3-msec square-wave clicks were presented with a 500 -msec onset-to-onset interstimulus interval and an 8-sec jittered intertrial interval (offset to onset 7-9 sec). Clicks were presented $50 \mathrm{~dB}$ above subjective hearing level, determined separately for each ear, and delivered via 5-m nonferromagnetic tubes. No task was involved, except that participants were asked to keep their eyes focused on a small fixation point throughout the measurement.

The MEG was recorded while participants were in a prone position, with a 148-channel magnetometer (MAGNES $2500 \mathrm{WH}, 4 \mathrm{D}$ Neuroimaging, San Diego, California). Data were continuously recorded with a sampling rate of $678.17 \mathrm{~Hz}$ and a bandpass filter of .1-200 Hz. For artifact control, the vertical and horizontal electrooculogram (from four electrodes near the left and right temporal canthus and above and below the right eye) was recorded with a SynAmps amplifier (Neuroscan Laboratories, Sterling, Virginia). The location of the nasion, left and right ear canal, and head shape of the participant were digitized with a Polhemus 3 Space Fasttrack before each session.

After noise reduction, trials with eye blinks were excluded from data analysis. Global noise was removed offline from MEG data by subtracting external, nonbiological noise recorded by 11 MEG reference channels. Before subtraction, reference channels were multiplied by individually calculated fixed-weight factors. This noisereduction procedure has little or no influence on biological signals, because of the distance from the reference sensors to the head of the participant (mean $=25.8 \mathrm{~cm}, \mathrm{SD}=6.00 \mathrm{~cm}$, range 15.5-36.5 $\mathrm{cm}$ ) relative to the distance between the head and adjacent sensors. Epochs of $100 \mathrm{msec}$ baseline before and $400 \mathrm{msec}$ after each stimulus were identified from continuous recordings. Epochs with amplitude $>4000 \mathrm{fT}$ and/or temporal gradients $>2500 \mathrm{fT} /$ sample were rejected. On average 98 artifact-free trials/participant were available for control subjects, and 96 artifact-free trials/participant were available for patients, with no differences between patients and control subjects at either pre- or post-treatment sessions. Artifact-free epochs were averaged and filtered with a $3-\mathrm{Hz}(12 \mathrm{~dB} /$ octave, zero-phase-shift) to $45-\mathrm{Hz}$ ( $24 \mathrm{~dB} /$ octave, zero-phase-shift) bandpass filter. Data processing used BESA 5.2 (http://www.besa. de) was applied to each participant individually.

The M50 was identified from the MEG event-related field (ERF) within a time window $40-80 \mathrm{msec}$ after stimulus onset as the largest amplitude before M100 (Figure 1), which was clearly evident in every participant. Visual inspection of the ERF confirmed auditory cortical activation, topographic distribution with ingoing and outgoing magnetic fields suggesting dipolar sources, and corresponding polarity reversal and topographic distribution opposite in direction to that of $\mathrm{M} 100$. As illustrated in Figure 1, a pair of regional dipoles were simultaneously fitted in the left and right hemisphere for a 20-msec interval around the S1 M50 peak. This latency was also used to obtain the M50 source strength in response to S2. Dipole fitting used information from all 148 magnetometers, as simulation (http://www.besa.de/updates/tools) indicated that 10\%-12\% of the variance in the measured signal was explained by activity at sensors over the opposite hemisphere. Only solutions exceeding at
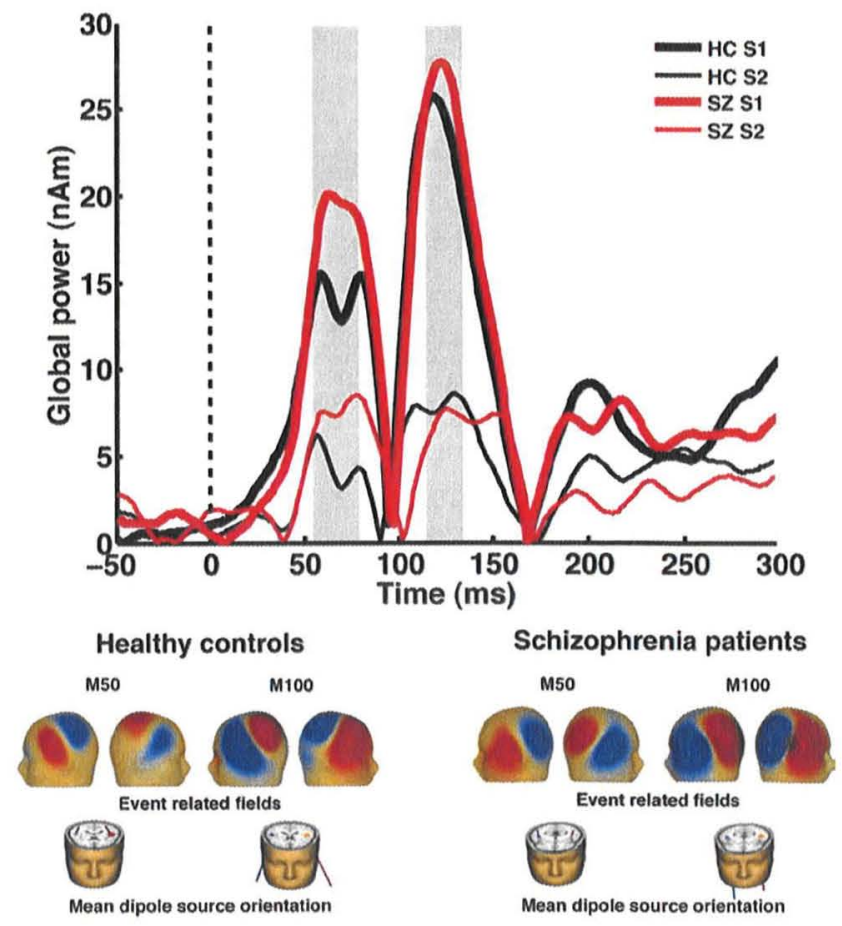

Figure 1. Top: grand-average source waveforms of the auditory brain response after S1 (thick line) and after S2 (thin line) averaged across participants within diagnostic group before treatment assignment. Bottom: topographic maps of the in- (red) and out-going (blue) magnetic fields for M50 (left) at $57.5 \mathrm{msec}$ and $\mathrm{M} 100$ (right) at $119 \mathrm{msec}$ averaged across participants within diagnostic group before treatment assignment. Locations of M50 regional dipoles averaged across participants are illustrated below magnetic field maps. Left: average location of M50 (Talairach coordinates $x, y, z$ left $-61.8,-16.3,21.6$; right $43.9,-3.1,27.9$ ) and $M 100$ (left $-46.3,-12.3$ 17.4 ; right $33.9,-4.9,26.2$ ) regional dipoles in healthy control subjects $(\mathrm{HC})$ right: average location of M50 (Talairach coordinates $x, y, z$ : left -39.1 , $-26.6,-10.0$ right $44.9,-22.9,-.3$ ) and M100 (left $-32.6,-17.9,-3.9$ right $45.7,-17.2,1.8$ ) regional dipoles in schizophrenia patients (SZ). 
least $75 \%$ goodness of fit were considered for analysis. To examine the neural specificity of effects, M100 was analyzed as the segment of the ERF with largest amplitude approximately $100 \mathrm{msec}$ after stimulus onset and dipole orientation opposite to that of M50 (Figure 1).

Auditory sensory gating was defined as a ratio of the peak amplitude (a single time point in the M50 latency window with the best goodness of fit expressed in $\mathrm{nAm}$ ) of the $\mathrm{S} 2$ response divided by the peak amplitude of the S1 response, each scored for left- and righthemisphere dipoles. Effects of disorder on gating ratios were evaluated by comparing the pretreatment measures in a Group (patient, control) $\times$ Hemisphere analysis of variance (ANOVA). Effects of treatment on gating ratios of patients were examined in Treatment $(\mathrm{CE}$, Cogpack) $\times$ Time (pre-/post-treatment) $\times$ Hemisphere ANOVAs. Significant main effects and interactions were decomposed by simple-effects ANOVAs or $t$ tests. Some ratio findings were also explored by examining separate S1- and S2-evoked responses. The M50 and M100 peak latencies did not bear on present hypotheses and will not be considered further. Before treatment, patients (mean \pm SD: $58.4 \pm 14.3 \mathrm{msec}$ ) and control subjects $(58.7 \pm 15.7$ msec) had similar M50 peak latencies ( $F<1)$; neither M50 nor M100 latencies differed as a function of treatment (Time and Group $\times$ Time $F<1)$ or patient group before $(F<1)$ or after $(F<1)$ treatment. Patients (97.4 $\pm 20.4 \mathrm{msec})$ and control subjects $(101.7 \pm 15.6 \mathrm{msec})$ had similar M100 peak latencies $(t<1)$.

Effects of treatment on overt cognitive performance were evaluated in a Treatment $\times$ Time ANOVA for each of the three VMLT scores (immediate recall, working memory, delayed recall after distraction) and mean word fluency score (percentile rank on the RWT). Relationships among gating ratios (left- and right-hemisphere, before and after treatment), cognitive test scores, and clinical measures (BPRS, GAF before and after treatment) were explored with correlations.

\section{Results}

\section{Pretreatment Auditory Gating in Schizophrenia}

Patients (mean \pm SD: $.53 \pm .2$ ) had higher gating ratios than control subjects $[.38 \pm .1 ; F(1,65)=15.14, p<.0002]$ (Figure 2) with no difference between hemispheres (Hemisphere, $F<1$; Group $X$ Hemisphere, $F<1$ ). This pretreatment group gating difference resulted from the second click: S1 M50 did not differ by group $[t(65)=$ $-.65]$, whereas S2 M50 was larger in patients $(18.0 \pm 13.2 \mathrm{nAm})$ than in

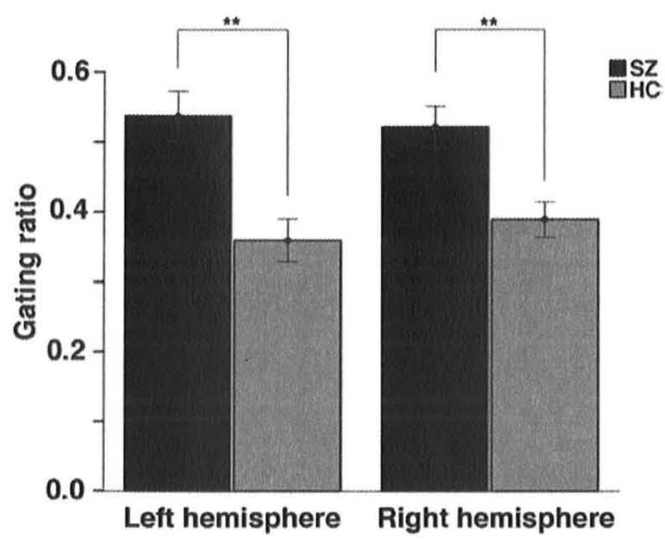

Figure 2. Gating ratios (ratio of $\mathrm{S} 2 / \mathrm{S} 1$ dipole activity in $\mathrm{nAm} / \mathrm{cm}^{2}$, ordinate) of healthy control subjects $(\mathrm{HC})$ and schizophrenia patients (SZ) before assignment of patients to treatment. Bars indicate means \pm SEM. ${ }^{*}$ Significant group differences: $p<.01$.

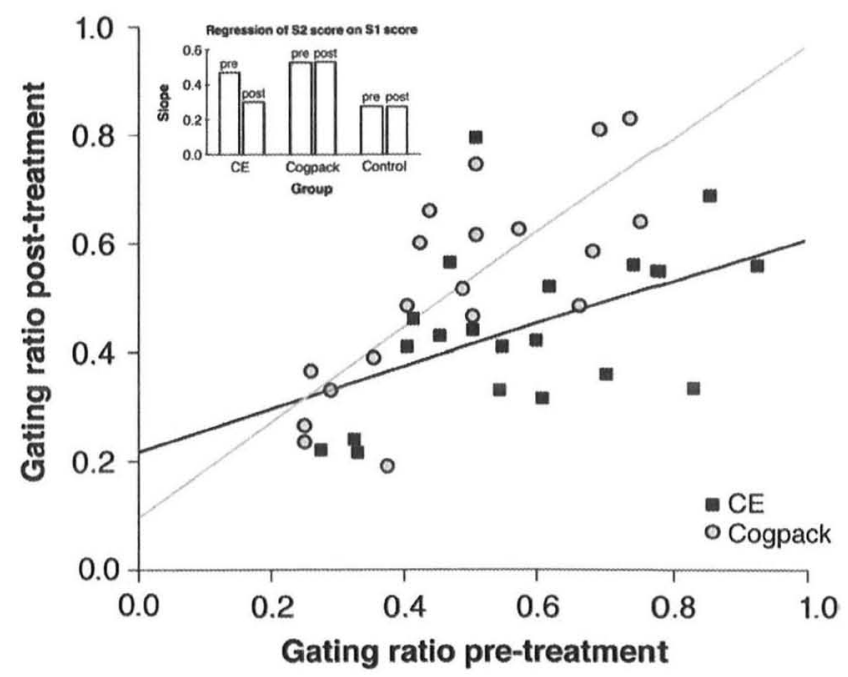

Figure 3. Relationship between gating ratios plotted for patients with respect to treatment, each symbol representing a single participant. The scatterplot illustrates the relationship of gating ratio before (abscissa) and after (ordinate) treatment. The bar graph (inset) unpacks the gating-ratio slope findings in terms of separate S1 and S2 amplitudes, separately before and after treatment. CE, Cognitive Exercises; Cogpack, Cognitive Package.

control subjects [11.5 $\pm 7.5 \mathrm{nAm}, t(65)=-2.4, p<.02]$. Before treatment there was no significant gating difference between patients later assigned to CE versus Cogpack $(t<1)$. Patients and control subjects did not differ in S1 M100 or S2 M100 amplitude or M100 gating ratio (all $t<1$ ).

\section{Treatment Effects on Auditory Gating}

Figure 3 provides a scatterplot of pre- versus post-treatment gating ratios for patients as a function of type of treatment. Gating ratios decreased (i.e., improved) after treatment but significantly so only after CE [Treatment $F<1$; Time, $F(1,37)=4.12, p<.05$ Treatment $\times$ Time $F(1,37)=11.97, p<.002$; no interactions with Hemisphere]. Post hoc analyses confirmed the gating ratio reduction after $C E[$ Time, $F(1,19)=11.52, p<.003]$ but not after Cogpack $[$ Time, $F(1,18)=1.6, p=.23]$. Effects sizes for the latter two comparisons are shown in the left portion of Figure 4.

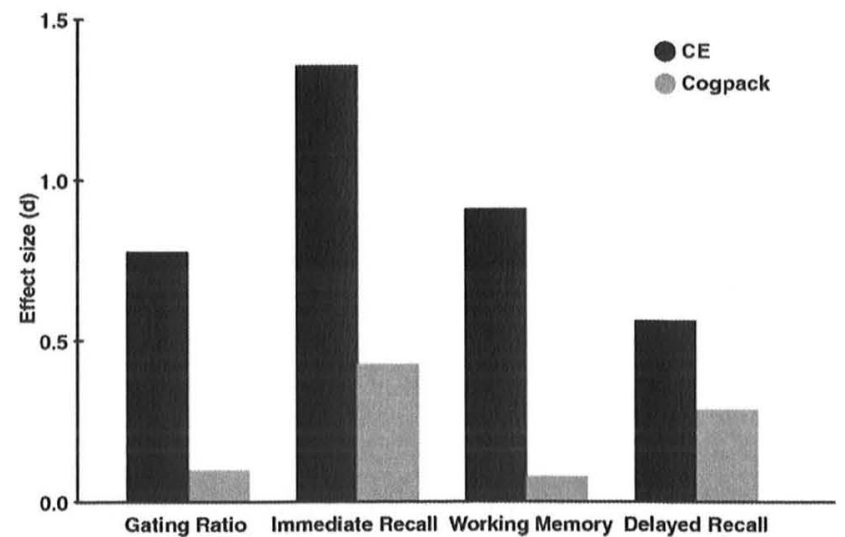

Figure 4. Effect sizes (Cohen's $d$ in SD units, ordinate) for change from pretreatment to post-treatment in M50 gating ratio and verbal memory test performance (immediate recall, working memory, and delayed recall) for the Cognitive Exercises (CE; black bars) and Cognitive Package (Cogpack; gray bars) patient groups. 
The CE normalized gating ratio of patients; Cogpack did not. At the pretreatment assessment, gating ratio of both patient groups exceeded that of control subjects [CE vs. control: $t(46)=4.41, p<$ .001 , Cogpack vs. control, $t(45)=2.55, p<.02]$. After $C E$, ratio of patients $(.44 \pm .15)$ no longer differed from that of control subjects $(.40 \pm .13, t<1)$, whereas after Cogpack ratio of patients $(.52 \pm .19)$ still differed $[t(32)=-.2 .09, p<.05]$. Type of medication did not influence gating ratio before or after treatment.

The differential effect of type of cognitive treatment on mean M50 gating ratio was specific both in the aspect of the gating ratio affected and in the MEG component affected. In further dissection of the Treatment $\times$ Time interaction for M50 gating ratio, S1 M50 did not vary across time with type of treatment. The S2 M50 was reduced in the CE group $[F(1,19)=15.29, p<.001]$ but not in the Cogpack group $(F<1)$. The treatment effect was specific to M50: there were no differences between treatment groups in $\mathrm{M} 100 \mathrm{am}$ plitude or ratio. An ANOVA adding the within-subject factor Component (M50, M100) confirmed larger changes for M50 than for M100 ratio after CE [Treatment, $F<1$; Time, $F(1,31)=1.15, p<.29$; Component, $F(1,36)=36.98, p<.0001$; Treatment $\times$ Time, $F<1$; Treatment $\times$ Component, $F<1$; Time $\times$ Component, $F<1.5$; Treatment $\times$ Time $\times$ Component, $F(1,36)=10.49, p<.003]$.

The treatment groups differed in slope of the regression of post-treatment on pretreatment gating ratio, separate from differential treatment effects on mean gating ratio, illustrated in Figure 3. A difference only in mean treatment effects would appear as a group difference in intercept. The difference in slopes indicates that CE patients who were the worst gaters before treatment benefited more from treatment than was the case for such Cogpack patients. The upper left insert in Figure 3 unpacks the gating-ratio slope findings in terms of separate S1 and S2 amplitudes before and after treatment. With treatment the relationship in the CE group changed to match that of control subjects. No such effect was evident for the Cogpack group. A test for homogeneity of regression slopes in the scatterplot showed this to be a reliable difference $(p=.05)$. An alternative test (32) based on hierarchical regression provided marginal support $(p=.08)$. These tests vary in statistical assumptions, and this finding should be taken as tentative.

\section{Treatment Effects on Verbal Learning and Memory}

Cognitive test scores both before and after treatment were available for $17 \mathrm{CE}$ and 18 Cogpack patients. Figure 5 illustrates changes in test performance in the two treatment groups, and Figure 4 illustrates substantial differences in effect size for the two types of treatment. Patients generally improved, more so after CE. Immediate recall improved more after $C E$ than after Cogpack [Time, $F(1,33)=32.14, p<.0001$; Treatment $\times$ Time, $F(1,33)=5.99, p=$ .02 ; simple main effect of Time for $C E, F(1,16)=31.39, p<.0001$; for Cogpack, $F(1,17)=5.44, p<.04]$. Similar results were obtained for working memory $[$ Treatment $\times$ Time, $F(1,33)=6.32, p<.02$; Time for $C E, F(1,16)=16.82, p<.001$; for Cogpack, $F<1]$. Delayed recall improved similarly across patient groups $[F(1,33)=16.11, p<.001]$ without differences by treatment (Treatment $\times$ Time, $F<1$ ). No effects of treatment were found on verbal fluency (main effect and interaction $p>.19$, simple comparisons $p=\mathrm{ns}$ ). Before treatment (higher, poorer) M50 gating ratio varied with (lower) immediate recall $[r(33)=-.36, p<.03]$ in the patient sample. This relationship remained after treatment $[r(33)=-.33, p=.05]$, with no difference between the treatment groups. With CE treatment, larger reduction (improvement) in left-hemisphere ratio was related to more improvement in immediate recall $[r(17)=.47, p=.056]$, and larger reduction in right-hemisphere ratio was related to more improvement in working memory $[r(16)=.50, p<.05]$. These correlations

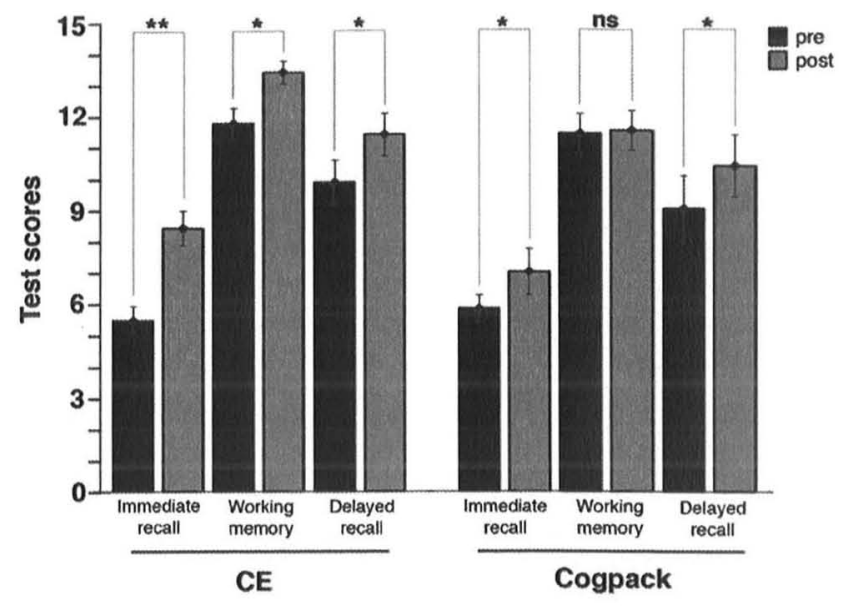

Figure 5. Performance of patients before and after treatment with Cognitive Exercises (CE) or Cognitive Package (Cogpack). Performance is represented separately for the three subtests of the Verbaler Lern und Merkfähigkeitstest. Ordinate denotes the number of items recalled from the 15-item word list of the Verbaler Lern und Merkfähigkeitstest. Immediate recall: number of words reproduced immediately after presentation; working memory: number of words recalled after word list repetition (maximum five repetitions); delayed recall: number of words recalled $30 \mathrm{~min}$ after training with the intermission including distraction by another word list. Statistica effects are marked by asterisks: ${ }^{*} p<.05 ;{ }^{* *} p<.01$; ns denotes nonsignificant differences between pre- and post-treatment.

were smaller and nonsignificant in the Cogpack group. The treatment groups did not differ in these correlations, so this additional evidence of effectiveness of CE cannot be interpreted with confidence. There was no correlation between age and gating ratio or test performance measures before or after intervention.

\section{Clinical Improvement}

IIIness status of patients improved [GAF, $F(1,30)=12.71, p<$ $.001 ; B P R S, F(1,30)=18.51, p<.001]$ irrespective of type of treatment (interactions $F<1$ ). Change in these scores did not vary with change in M50 or M100 gating ratio.

\section{Discussion}

The present study confirmed the previously reported impaired auditory sensory gating in schizophrenia patients $(6,16)$ : before treatment, higher gating ratios were found in patients than in control subjects. Group differences were confined to M50 (not M100) and to the S2-evoked M50 (not S1-evoked M50). This supports the hypothesis of a gating deficit in schizophrenia as deficient filtering of redundant sensory information and not because of deficient information encoding.

\section{Treatment Effects on Auditory Gating}

The CE treatment, specifically targeting discrimination ability in the auditory/verbal system, normalized auditory gating. The specificity of the benefit of CE treatment was demonstrated over a nonspecific cognitive treatment of equal (4-week) duration and subjective effort. Changes in gating ratio were due to changes in M50 attenuation to $\mathrm{S} 2$, indicating improvement in filtering of redundant information, rather than to changes in S1-evoked M50, which would have indicated a change in stimulus encoding. These results indicate that $\mathrm{CE}$ improved discrimination accuracy in the auditory system and, as a consequence, discrimination of signal processing Beneficial effects of CE might have been exerted by compensatory processes such as attention and working memory, which have been 
shown to vary with $\mathrm{P} 50$ gating ratios in healthy individuals and schizophrenia patients $(17,33)$, but these effects should have been comparable for CE and Cogpack, whereas the focus on auditoryverbal discrimination accuracy is specific to $C E$.

Although this initial study was not designed to identify mechanisms of action, results are in accord with a prominent model of sensory gating. Poor sensory gating has been discussed because of insufficient suppression of S2 relative to $\mathrm{S} 1$ response $(34,35)$ or instead as a product of abnormal S1 amplitude compared with normal S2 response (36-38). Present results support the former view, because both pretreatment abnormality and treatment benefit were confined to $\mathrm{S} 2$.

Treatment-induced neuroplastic changes within primary auditory cortex might be modeled as follows: in the healthy brain, the processing of $\mathrm{S} 1$ involves a substantial portion of the entire auditory network, resulting in the P50/M50 component. While this network is still engaged in processing and/or transferring S1-related information, S2 is presented but does not engage similar network activity due to recurrent inhibitory mechanisms activated by S1 (34). These inhibitory or refractory processes should result in an attenuated M50 to S2. If less organized neuronal networks are assumed for schizophrenia, S1 would not activate as much of the network, including inhibitory mechanisms. As a consequence, more of the network might be activated by S2. Studies of the mammalian auditory system support this account: enhancement and degradation of neuronal response selectivity can be affected by manipulating acoustic experience in early postnatal life (39), and deficits in temporal processing induced in auditory cortex during infancy can be repaired by intensive treatment during young adulthood (40).

\section{Auditory Gating and Cognitive Function}

The CE also differentially improved overt performance in immediate recall and working memory, paralleling the M50 gating-ratio changes. Thus, lower-level auditory processing indexed in M50 varied with higher-level verbal learning and memory. Impaired verbal learning and memory are characteristic deficits in schizophrenia. Such measures correlate with M50 gating ratio (41) and might constitute a core element of impaired cognitive function. Similar effects of $C E$ on verbal learning and memory have been reported $(8,13)$. Together, these results support the hypothesis that increased efficiency in elemental processes fosters higher-order cognition $(9,10)$.

By demonstrating that, with an appropriately targeted intervention, beneficial effects on overt performance can be obtained in as few as 20 sessions, the present study builds on previous studies reporting success after $50-100$ sessions $(8,13,14)$. Longer CE treatment might augment present effects: Fisher et al. (8) documented larger effect sizes after 100 than after 50 treatment sessions, and a meta-analysis (2) suggests that duration of treatment matters. Present effect sizes (Figure 4) suggest (slight) positive changes after Cogpack, supporting the beneficial effects of a broad intervention but more substantial effects on cortical and test performance after $\mathrm{CE}$. These results underscore the value of targeting relevant neuroplasticity in treatment development (12).

\section{Symptom Measures}

Whereas global measures of symptom severity indicated improvement as a function of treatment, changes across time were not significantly modulated by the type of treatment. This is in line with studies that also did not find effects of auditory training on general psychopathology $(8,14)$. Whether training can affect traditional clinical symptoms remains to be determined, although a relationship between negative symptoms and right-hemisphere M50 gating ratio has been reported (20). Moreover, beneficial effects of training on symptoms might show up only after extended follow-up. This remains to be evaluated in a prospective study.

\section{Limitations}

Limitations of the present study can be noted. First, all patients were taking medication. The recruitment setting did not allow the study of unmedicated patients. However, because there were no differences in medication between treatment groups, a confound of medication with treatment type is unlikely. Second, the total computer-session treatment time was somewhat (not dramatically) less for Cogpack than for CE. However, effort regarding frequency and duration of training sessions rather than total minutes of training time was matched in this study. Furthermore, the schedules for the two treatment methods reflect their normal use in the field and spanned the same 4-week period. Third, this initial study did not explore dose-response relationships involving, for example, duration of CE or Cogpack treatment. Finally, conclusions regarding the generalization of treatment effects on higher-order cognitive function should be substantiated in future studies by comparing treatment effects with a broader neuropsychological test battery than employed in the present study. Nevertheless, available results indicate that CE has promise for cognitive and neural remediation in schizophrenia.

This work was supported by the German Research Foundation (Deutsche Forschungsgemeinschaft, DFG; Ro805/14). We thank Sarah Schulz, Petia Popova, and Ursula Lommen for their assistance in data collection and analysis and Dr. Patrick Berg and Dr. Christian Wienbruch for consultation on MEG methodology. Michael M. Merzenich is an executive officer of PositScience, which created the original Englishlanguage version of the training software employed in the present trial. All other authors report no biomedical financial interests or potential conflicts of interest.

ClinicalTrials.gov: Training-Induced Cerebral Reorganization in Schizophrenia; http://www.clinicaltrials.gov/; NCT00695708.

Supplementary material cited in this article is available online.

1. Gold JM (2004): Cognitive deficits as treatment targets in schizophrenia. Schizophr Res 72:21-28.

2. Silverstein SM, Wilkniss SM (2004): At issue: The future of cognitive rehabilitation of schizophrenia. Schizophr Bull 30:679-692.

3. Hayes RL, McGrath JJ (2000): Cognitive rehabilitation for people with schizophrenia and related conditions. Cochrane Database Syst Rev CD000968.

4. McGurk SR, Twamley EW, Sitzer DI, McHugo GJ, Mueser KT (2007): A meta-analysis of cognitive remediation in schizophrenia. Am J Psychiatry 164:1791-1802.

5. Twamley EW, Jeste DV, Bellack AS (2003): A review of cognitive training in schizophrenia. Schizophr Bull 29:359-382.

6. Heinrichs RW (2004): Meta-analysis and the science of schizophrenia: Variant evidence or evidence of variants? Neurosci Biobehav Rev 28:379394.

7. Albus M, Hubmann W, Mohr F, Hecht S, Hinterberger-Weber P, Seitz NN, Küchenhoff $H$ (2006): Neurocognitive functioning in patients with firstepisode schizophrenia: Results of a prospective 5-year follow-up study. Eur Arch Psychiatry Clin Neurosci 256:442-451.

8. Fisher M, Holland C, Merzenich MM, Vinogradov S (2009): Using neuroplasticity-based auditory training to improve verbal memory in schizophrenia. Am J Psychiatry 166:805-811.

9. Merzenich MM (2001): Cortical plasticity contributing to child development. In: McClelland J, Siegler R, Rahway N, editors. Mechanisms in Cognitive Development. Mahwah, New Jersey: Lawrence Erlbaum, 67-96.

10. Mahncke HW, Bronstone A, Merzenich MM (2006): Brain plasticity and functional losses in the aged: Scientific bases for a novel intervention. Prog Brain Res 157:81-109. 
11. Buonomano DV, Merzenich MM (1998): Cortical plasticity: From synapses to maps. Annu Rev Neurosci 21:149-186.

12. Elbert T, Rockstroh B (2004): Reorganization of human cerebral cortex: The range of changes following use and injury. Neuroscientist 10:129-141.

13. Adcock RA, Dale C, Fisher M, Aldebot S, Genevsky A, Simpson GV, et al. (2009): When top-down meets bottom-up: Auditory training enhances verbal memory in schizophrenia. Schizophr Bull 35:1132-1141.

14. Fisher M, Holland C, Subramaniam K, Vinogradov S (2009): Neuroplasticity-based cognitive training in schizophrenia: An interim report on the effects 6 months later. Schizophr Bull 36:869-879.

15. Smith GE, Housen P, Yaffe K, Ruff R, Kennison RF, Mahncke HW, Zelinski EM (2009): A cognitive training program based on principles of brain plasticity: Results from the Improvement in Memory with Plasticitybased Adaptive Cognitive Training (IMPACT) study. J Am Geriatr Soc 57:594-603.

16. Bramon E, Rabe-Hesketh S, Sham P, Murray RM, Frangou S (2004): Metaanalysis of the P300 and P50 waveforms in schizophrenia. Schizophr Res 70:315-329.

17. Yee CM, Williams TJ, White PM, Nuechterlein KH, Ames D, Subotnik KL. (2010): Attentional modulation of the P50 suppression deficit in recentonset and chronic schizophrenia. J Abnorm Psychol 119:31-39.

18. Winterer G, Ziller M, Dorn H, Frick K, Mulert C, Wuebben Y, et al. (2000): Schizophrenia: Reduced signal-to-noise ratio and impaired phase-locking during information processing. Clin Neurophysiol 111:837-849.

19. Potter D, Summerfelt A, Gold J, Buchanan RW (2006): Review of clinical correlates of P50 sensory gating abnormalities in patients with schizophrenia. Schizophr Bull 32:692-700.

20. Clementz BA, Dzau JR, Blumenfeld LD, Matthews S, Kissler J (2003): Ear of stimulation determines schizophrenia-normal brain activity differences in an auditory paired-stimuli paradigm. Eur J Neurosci 18:2853-2858.

21. Edgar JC, Huang MX, Weisend MP, Sherwood A, Miller GA, Adler LE, Cañive JM (2003): Interpreting abnormality: An EEG and MEG study of P50 and the auditory paired-stimulus paradigm. Biol Psychol 65:1-20.

22. Thoma RJ, Hanlon FM, Moses SN, Ricker D, Huang M, Edgar C, et al. (2005): M50 sensory gating predicts negative symptoms in schizophrenia. Schizophr Res 73:311-318.

23. Hsieh MH, Liu K, Liu SK, Chiu MJ, Hwu HG, Chen AC (2004): Memory impairment and auditory evoked potential gating deficit in schizophrenia. Psychiatry Res 130:161-169.

24. Olbrich R (1998): [Computer-assisted psychiatric rehabilitation]. Psychiatr Prax 25:103-104.

25. Geibel-Jakobs M, Olbrich R (1998): [Computer-assisted cognitive training of schizophrenic patients. Use of evaluation outcome for developing an individualized training method]. Psychiatr Prax 25:111-116.
26. Sartory G, Zorn C, Groetzinger G, Windgassen K (2005): Computerized cognitive remediation improves verbal learning and processing speed in schizophrenia. Schizophr Res 75:219-223.

27. Overall JE, Gorham DR (1962): The brief psychiatric rating scale. Psychol Rep 10:799-812.

28. Lukoff D, Liberman RP, Nuechterlein KH (1986): Symptom monitoring in the rehabilitation of schizophrenic patients. Schizophr Bull 12:578-602.

29. Ackenheil M, Stotz-Ingenlath G, Dietz-Bauer R, Vossen A (1999): M.I.N.I. Mini International Neuropsychiatric Interview, German Version 5.0.0 DSM IV. Munich, Germany: Psychiatrische Universitätsklinik München.

30. Helmstaedter L, Lux (2001): VLTM: Verbaler Lern- und Merkfähigkeitstest. Göttingen, Germany: Hogrefe.

31. Aschenbrenner S, Tucha O, Lange KW (2000): Regensburg Word Fluency Test [Regensburger Wortflüssigkeits-Test]. Göttingen, Germany: Hogrefe.

32. DeShon R, Alexander R (1996): Alternative procedures for testing regression slope homogeneity when group error variances are unequal Psychol Methods 3:261-277.

33. Lijffijt M, Lane SD, Meier SL, Boutros NN, Burroughs S, Steinberg لL, et al. (2009): P50, N100, and P200 sensory gating: Relationships with behavioral inhibition, attention, and working memory. Psychophysiology 46: 1059-1068.

34. Freedman R, Adler LE, Gerhardt GA, Waldo M, Baker N, Rose GM, et al. (1987): Neurobiological studies of sensory gating in schizophrenia. Schizophr Bull 13:669-678.

35. Clementz BA, Geyer MA, Braff DL (1997): P50 suppression among schizophrenia and normal comparison subjects: A methodological analysis. Biol Psychiatry 41:1035-1044.

36. Blumenfeld LD, Clementz BA (2001): Response to the first stimulus determines reduced auditory evoked response suppression in schizophrenia: Single trials analysis using MEG. Clin Neurophysiol 112:1650 1659.

37. Edgar JC, Hanlon FM, Huang MX, Weisend MP, Thoma RJ, Carpenter B, et al. (2008): Superior temporal gyrus spectral abnormalities in schizophrenia. Psychophysiology 45:812-824.

38. Brenner CA, Kieffaber PD, Clementz BA, Johannesen JK, Shekhar A, O'Donnell BF, et al. (2009): Event-related potential abnormalities in schizophrenia: A failure to "gate in" salient information? Schizophr Res 113:332-338.

39. Dahmen JC, King AJ (2007): Learning to hear: Plasticity of auditory cortical processing. Curr Opin Neurobiol 17:456-464.

40. Zhou X, Merzenich MM (2009): Developmentally degraded cortical temporal processing restored by training. Nat Neurosci 12:26-28.

41. Thoma RJ, Hanlon FM, Moses SN, Edgar JC, Huang M, Weisend MP, et al. (2003): Lateralization of auditory sensory gating and neuropsychological dysfunction in schizophrenia. Am J Psychiatry 160:1595-1605. 